
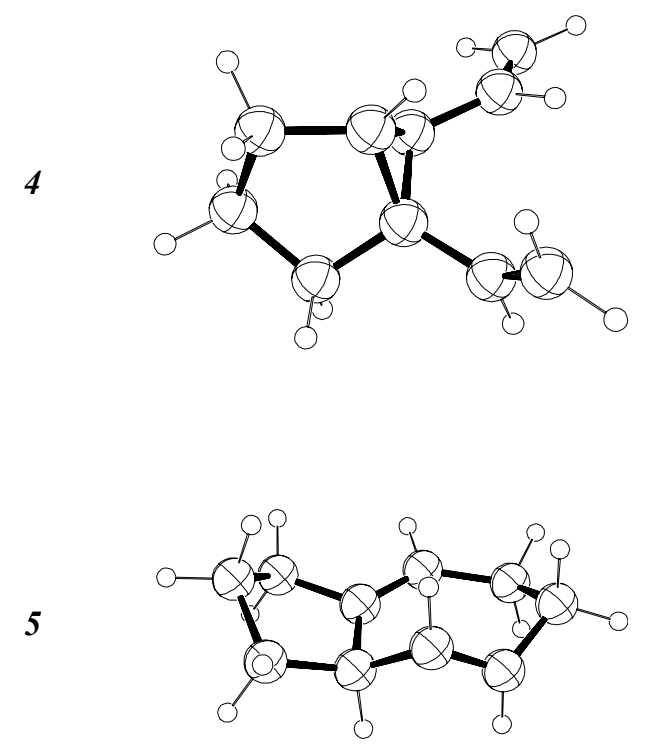

6

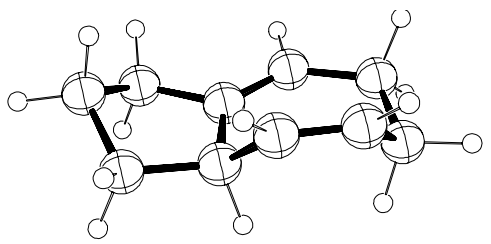

7

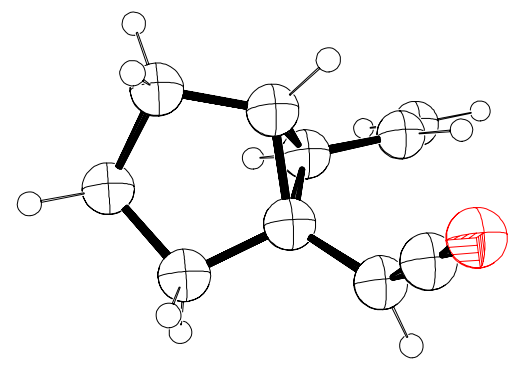

8

\section{B3LYP/6-31G**//HF=-389.433829, G=-21.8649}

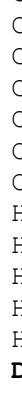

$\begin{array}{rrrr} & 0.000000 & 0.000000 & 0.000000 \\ & 4.940150 & 0.000000 & 0.000000 \\ & 4.131750 & 1.062430 & 0.000000 \\ & 3.302580 & 1.530830 & -1.143950 \\ 1.839990 & 1.074070 & -1.291840 \\ C & 1.275210 & 0.041860 & -0.397210 \\ & 2.895280 & 0.679930 & -2.320590 \\ & 3.162750 & -0.372210 & -2.370800 \\ & 5.519970 & -0.272240 & 0.877090 \\ & 5.050490 & -0.633670 & -0.876410 \\ H & 4.064370 & 1.673130 & 0.902360 \\ & 1.967160 & -0.724620 & -0.051410\end{array}$

Divinylcyclopropane trans product

B3LYP $/ 6-31 \mathrm{G} * \star / / \mathrm{HF}=-389.422687, \mathrm{G}=-20$

C $0.000000=0.000000=0.000000$

C $1.5756600 .000000 \quad 0.000000$

C $1.8722201 .465060 \quad 0.000000$

C $1.747850 \quad 2.117900-1.165540$

C $-0.569630 \quad 2.170630-1.447290$

C $-0.744120 \quad 0.907900-0.990880$

$\mathrm{H}-0.361210-1.025120-0.154100$

$\mathrm{H}-0.314370 \quad 0.271580 \quad 1.017790$

$\mathrm{H} \quad 1.943590-0.484980-0.911100$

$\mathrm{H} \quad 1.937150 \quad-0.566600 \quad 0.866610$

$\mathrm{H} \quad 1.573950 \quad 1.992710 \quad 0.910140$

H $\quad 1.960050 \quad 1.582190-2.092930$

Divinylcyclopropane cis product

B3LYP / 6-31G * $/ / \mathrm{HF}=-389.468748, \mathrm{G}=-20.7467$

$\begin{array}{lrrrrrrr}\mathrm{C} & 0.000000 & 0.000000 & 0.000000 & \mathrm{H} & -0.331300 & 2.764960 & -0.684830 \\ \mathrm{C} & 1.542690 & 0.000000 & 0.000000 & \mathrm{C} & 0.191060 & 2.923630 & 0.270600 \\ \mathrm{C} & 2.222370 & 1.349840 & 0.000000 & \mathrm{H} & -0.900750 & 2.555820 & 2.192110 \\ \mathrm{C} & 1.680140 & 2.569250 & 0.094810 & \mathrm{C} & 2.482690 & 3.856340 & 0.159930 \\ \mathrm{C} & -0.489210 & 2.058130 & 1.314690 & \mathrm{H} & 3.503390 & 3.701150 & 0.523470 \\ \mathrm{C} & -0.588940 & 0.732150 & 1.183940 & \mathrm{H} & 2.557250 & 4.311720 & -0.839990 \\ \mathrm{H} & 1.900800 & -0.563960 & 0.875550 & \mathrm{C} & 1.625650 & 4.761240 & 1.062690 \\ \mathrm{H} & 1.895330 & -0.569880 & -0.872690 & \mathrm{H} & 1.757880 & 4.465370 & 2.111090 \\ \mathrm{H} & -0.351490 & -1.037300 & -0.020730 & \mathrm{H} & 1.877800 & 5.823410 & 0.981740 \\ \mathrm{H} & -0.349520 & 0.463900 & -0.933580 & \mathrm{C} & 0.190940 & 4.444470 & 0.600200 \\ \mathrm{H} & -1.080700 & 0.147150 & 1.959010 & \mathrm{H} & -0.034790 & 5.019830 & -0.305500 \\ \mathrm{H} & 3.311060 & 1.296070 & -0.061480 & \mathrm{H} & -0.569550 & 4.706790 & 1.343060 \\ \text { Ketene-substituted substrate } & & & & \end{array}$

\section{B3LYP / 6-31G**//HF =-463.437375, G=-22.9534}

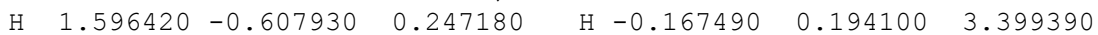

C $-0.634820-0.746250 \quad 0.199750 \quad$ H $\quad 1.203710 \quad-0.333560 \quad 2.439380$

$\mathrm{H}-1.374090-1.178710-0.470110 \quad \mathrm{H}-1.739790-1.034410 \quad 2.019150$

$\begin{array}{llllllll}\text { C } & -0.210850 & 0.698950 & 0.052990 & \text { C } & 0.982940 & -0.684730 & -1.825340\end{array}$

$\mathrm{H}-0.341590-2.106620 \quad 1.900850 \quad \mathrm{C}-2.053110 \quad 1.582610-1.322130$

C $-0.784820 \quad 1.633800 \quad-0.966840 \quad$ H $-0.177220 \quad 2.418370-1.409230$

C $0.731970-0.431660-0.390900 \quad 0 \quad-3.179350 \quad 1.506050-1.643210$

C $\quad 0.043210 \quad 1.240690 \quad 1.467410 \quad$ C $\quad 2.147430 \quad-1.104420 \quad-2.328210$

$\mathrm{H}-0.823180 \quad 1.848020 \quad 1.756160 \quad \mathrm{H} \quad 0.143950-0.509340-2.497080$

$\mathrm{H} \quad 0.926500 \quad 1.888130 \quad 1.523570 \quad \mathrm{H} \quad 3.011510-1.287360-1.693480$

C $0.160680-0.006250 \quad 2.375090 \quad$ H $\quad 2.275900-1.281100 \quad-3.391590$

C $-0.697170-1.0932201 .681680$

Ketene-substituted trans product

B3LYP $/ 6-31 \mathrm{G} * * / / \mathrm{HF}=-463.437328, \mathrm{G}=-21.6821$

C $0.000000 \quad 0.000000 \quad 0.000000 \quad$ C $-0.665940 \quad 0.692950-1.149950$

$\begin{array}{llllllll}\text { C } & 1.341520 & 0.000000 & 0.000000 & \text { H } & -0.121370 & 0.476650 & -2.079040\end{array}$

C $\quad 0.555720 \quad 2.711810 \quad 0.000000 \quad$ C $-2.201180 \quad 0.582710-1.370440$

C $-0.422450 \quad 2.172310-0.774720 \quad \mathrm{H}-2.410720 \quad 0.310890-2.409880$

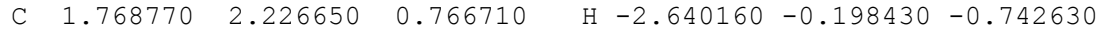

C $2.016790 \quad 0.728060 \quad 1.115160 \quad$ C $-2.782920 \quad 1.983320-1.047080$

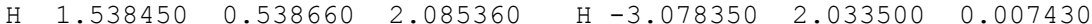

$\mathrm{H} \quad 3.094790 \quad 0.584810 \quad 1.226610 \quad \mathrm{H}-3.666200 \quad 2.225820-1.645120$

$\begin{array}{llllllll}0 & 2.563620 & 3.071080 & 1.148540 & \mathrm{C} & -1.609120 & 2.958050 & -1.286970\end{array}$

$\mathrm{H}-0.527920 \quad 0.059950 \quad 0.954260 \quad \mathrm{H}-1.492260 \quad 3.136560-2.366720$

$\mathrm{H} \quad 0.506980 \quad 3.794620 \quad 0.118710 \quad \mathrm{H}-1.731070 \quad 3.929320 \quad-0.799820$

H $\quad 1.857410 \quad 0.025350-0.962070$ 
\# Ball-and-stick figure

9

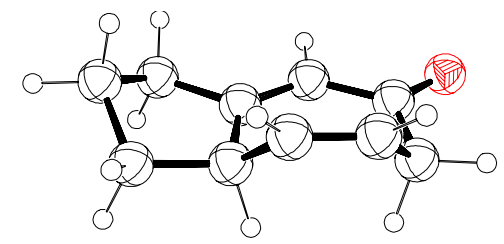

10

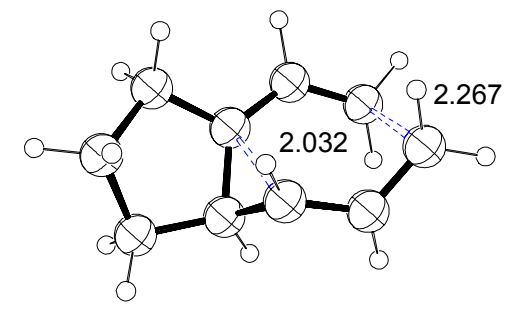

11

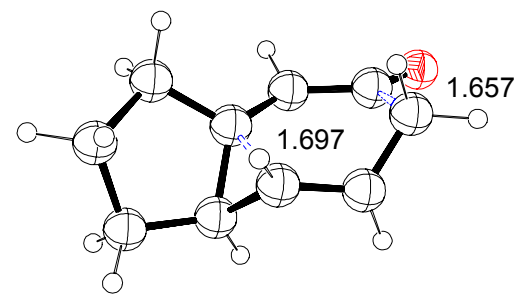

12



13

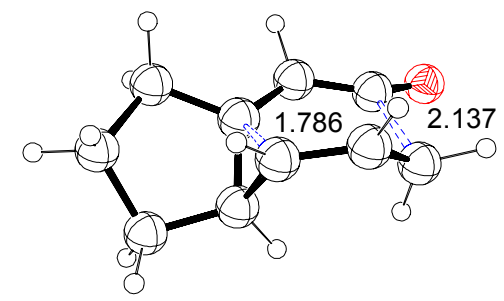

Level of theory $/$ basis $/ / \mathrm{HF}$ (hartree), $\Delta \mathrm{G}$ correction ( $\mathrm{kcal} / \mathrm{mol}$ ) from calc. real frequencies; $\lambda_{1}, \lambda_{2}$ (two lowest eigenvalues, transition structures)

\begin{tabular}{|c|c|c|c|c|c|c|c|}
\hline \multicolumn{8}{|c|}{ Ketene-substituted cis product } \\
\hline \multicolumn{8}{|c|}{$\mathrm{B} 3 L \mathrm{YP} / 6-31 \mathrm{G} * \star / / \mathrm{HF}=-463.48486, \mathrm{G}=-21.5273$} \\
\hline 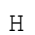 & -1.328720 & 0.850920 & 0.602720 & $\mathrm{H}$ & 2.20 & - & 1. \\
\hline 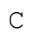 & -0 & & & $\mathrm{H}$ & & & \\
\hline$C$ & -1 & -0 . & -1. & $\mathrm{C}$ & -0 . & & \\
\hline $\mathrm{C}$ & -1.1 & -1.2 & & $\mathrm{H}$ & -1 . & 70 & 30 \\
\hline $\mathrm{C}$ & & & & $\mathrm{H}$ & & & \\
\hline $\mathrm{H}$ & -1 . & -1 & & 0 & & & \\
\hline $\mathrm{H}$ & -1 & -0 . & & $\mathrm{C}$ & -0 . & & \\
\hline C & & -1 & & $\mathrm{H}$ & -1 . & & \\
\hline $\mathrm{H}$ & 0 & -2 & & $\mathrm{C}$ & & & \\
\hline $\mathrm{H}$ & 0.1 & -2 & & $\mathrm{H}$ & -0. & & -2 \\
\hline $\mathrm{C}$ & 1.160560 & -0.776910 & 1.652630 & $\mathrm{H}$ & -0.862780 & 2.403610 & -1.020600 \\
\hline & & & & & & & \\
\hline
\end{tabular}

Divinylcyclopropane trans transition state (UB3LYP->closed shell)

B3LYP / 6-31G**//HF=-389.38608, G=-20.555; $\lambda=-409.73,137.01$

C $0.000000 \quad 0.000000 \quad 0.000000 \quad$ H $-0.632020-0.102250-2.016040$

C $2.267350 \quad 0.000000 \quad 0.000000$ C $0.767420 \quad 3.027140-1.070650$

$\begin{array}{llllllll}\text { C } & 2.272660 & 1.387890 & 0.000000 & \text { H } & 0.486360 & 3.248310 & -0.037200\end{array}$

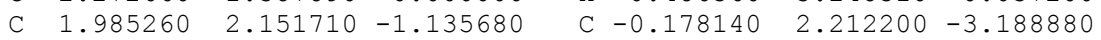

C $\quad 0.064180 \quad 1.848680-1.723120$ H $0.118150 \quad 1.421130-3.887080$

$\begin{array}{llllllll}\mathrm{C} & -0.277700 & 0.580160 & -1.241710 & \mathrm{H} & -1.259490 & 2.359540 & -3.324040\end{array}$

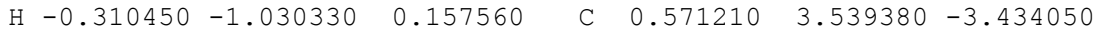

$\mathrm{H} \quad 0.041160 \quad 0.592340 \quad 0.904580 \quad \mathrm{H} \quad 0.077750 \quad 4.171050 \quad-4.178770$

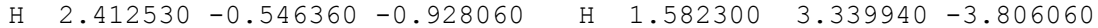

H $\quad 2.468410 \quad-0.562730 \quad 0.908730 \quad$ C $\quad 0.641940 \quad 4.203160 \quad-2.043140$

$\mathrm{H} \quad 2.060320 \quad 1.864010 \quad 0.961210 \quad \mathrm{H}-0.289870 \quad 4.743650-1.833920$

$\mathrm{H} \quad 2.410490 \quad 1.910070-2.104950 \quad \mathrm{H} \quad 1.468680 \quad 4.914770-1.949210$

Ketene-substituted trans transition state (UB3LYP->closed shell)

B3LYP / 6-31G**//HF=-463.407608, G=-20.9727; $\lambda=-242.2,94.81$

$\begin{array}{lrrrrrrr}\mathrm{C} & 0.000000 & 0.000000 & 0.000000 & \mathrm{C} & -0.719670 & 0.492520 & -1.191820 \\ \mathrm{C} & 1.419670 & 0.000000 & 0.000000 & \mathrm{H} & -0.115360 & 0.577540 & -2.093440 \\ \mathrm{C} & 0.575170 & 2.508830 & 0.000000 & \mathrm{C} & -2.214350 & 0.249610 & -1.340600 \\ \mathrm{C} & -0.520690 & 1.609100 & -0.142330 & \mathrm{H} & -2.577730 & 0.819040 & -2.204100 \\ \mathrm{C} & 1.909170 & 2.211010 & 0.415420 & \mathrm{H} & -2.460270 & -0.803240 & -1.515870 \\ \mathrm{C} & 2.082960 & 0.698180 & 1.069220 & \mathrm{C} & -2.839330 & 0.794380 & -0.034220 \\ \mathrm{H} & 1.575790 & 0.664570 & 2.035750 & \mathrm{H} & -2.852870 & 0.012140 & 0.733230 \\ \mathrm{H} & 3.158580 & 0.566770 & 1.169180 & \mathrm{H} & -3.875650 & 1.115090 & -0.173290 \\ \mathrm{O} & 2.913360 & 2.906430 & 0.377870 & \mathrm{C} & -1.911590 & 1.946540 & 0.403650 \\ \mathrm{H} & -0.539130 & -0.410710 & 0.850490 & \mathrm{H} & -2.223430 & 2.892910 & -0.059070 \\ \mathrm{H} & 0.331250 & 3.568440 & 0.071710 & \mathrm{H} & -1.911450 & 2.106920 & 1.486700\end{array}$
$\mathrm{H} \quad 1.952730-0.135740-0.939250$

Divinylcyclopropane cis transition state (UB3LYP->closed shell)

B3LYP / 6-31G**//HF=-389.4037, G=-20.5746; $\lambda=-423.56,144.24$

C $0.000000 \quad 0.000000 \quad 0.000000 \quad \mathrm{H} \quad 1.111740 \quad 1.006110-2.385590$

C $2.381940 \quad 0.000000 \quad 0.000000 \quad$ C $1.173660 \quad 2.011570-1.964000$

$\begin{array}{llllllll}\text { C } & 2.723180 & 1.343100 & 0.000000 & \mathrm{H} & -0.074590 & 3.337890 & -0.710970\end{array}$

C $2.2245802 .273610-0.917350$ C $2.750060 \quad 3.692150-1.085170$

C $\quad 0.215130 \quad 2.300440 \quad-0.845640 \quad \mathrm{H} \quad 2.742130 \quad 4.275260 \quad-0.157040$

$\begin{array}{lllllll}\text { C }-0.287400 & 1.354570 & 0.051280 & \text { H } & 3.799760 & 3.630590 & -1.404720\end{array}$

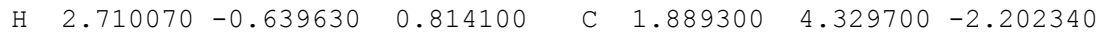

$\mathrm{H} \quad 2.194510-0.509330-0.935300 \quad \mathrm{H} \quad 1.074940 \quad 4.917490-1.766160$

$\mathrm{H}-0.339710-0.654570 \quad 0.797210 \quad \mathrm{H} \quad 2.464400 \quad 5.013380 \quad-2.833710$

H $\quad 0.161120-0.487260-0.952560 \quad$ C $\quad 1.320310 \quad 3.132140-3.000270$

$\mathrm{H}-0.771450 \quad 1.745040 \quad 0.946290 \quad \mathrm{H} \quad 2.036530 \quad 2.814290 \quad-3.767660$

$\mathrm{H} \quad 3.265940 \quad 1.743370 \quad 0.857430 \quad \mathrm{H} \quad 0.377360 \quad 3.369110 \quad-3.504810$

Ketene-substituted cis transition state (UB3LYP->closed shell)

B3LYP / 6-31G**//HF $=-463.410916, G=-21.4128 ; \lambda=-571.85,78.58$

$\begin{array}{llllllll}0 & 0.000000 & 0.000000 & 0.000000 & \text { C } & 4.828670 & -1.302170 & -0.167600\end{array}$

C $1.191140 \quad 0.000000 \quad 0.000000 \quad$ H $\quad 4.564270-2.230830-0.689620$

C $1.802110 \quad 2.048210 \quad 0.000000 \quad$ H $\quad 4.982990 \quad-1.560970 \quad 0.885730$

H $\quad 0.970410 \quad 2.544260 \quad 0.487970$ C $\quad 6.066140-0.653070-0.824280$

$\mathrm{H} \quad 1.688850 \quad 1.922600-1.076100 \quad \mathrm{H} \quad 6.640430-0.089230-0.081870$

C $\quad 3.063880 \quad 2.076400 \quad 0.555670$ H $\quad 6.747870 \quad-1.394580-1.250220$

H $3.184870 \quad 2.494340 \quad 1.553960$ C $5.488390 \quad 0.300650-1.897270$

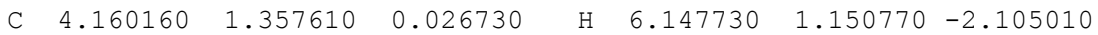

H $\quad 5.113930 \quad 1.422290 \quad 0.539270$ H $\quad 5.332390 \quad-0.234750-2.841530$

C $3.680300-0.315720-0.374460$ C $4.129650 \quad 0.726150-1.341810$

C $2.345940-0.735350-0.055030$ H $3.419630 \quad 1.145690-2.053980$ 





\# Ball-and-stick figure

$17 a-c i s$

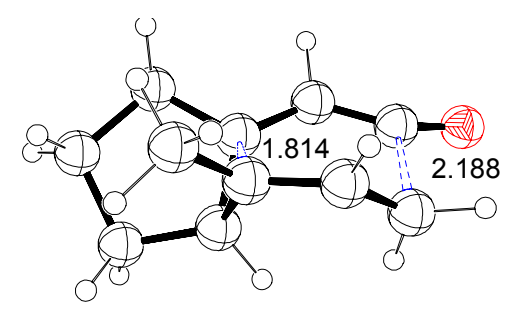

$17 a-$

trans

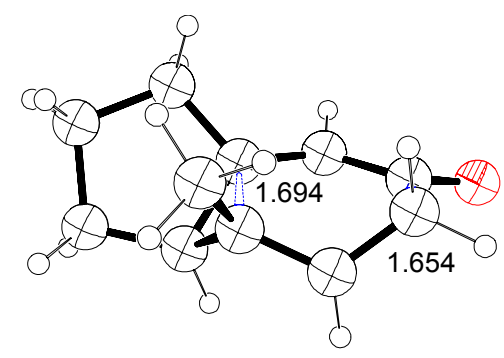

$17 b$-cis



$17 b-$ trans
Level of theory $/$ basis//HF (hartree), $\Delta \mathrm{G}$ correction ( $\mathrm{kcal} / \mathrm{mol}$ ) from calc. real frequencies; $\lambda_{1}, \lambda_{2}$ (two lowest eigenvalues, transition structures)

\section{1-substituted ketene cis transition state
$\mathrm{B} 3 \mathrm{LYP} / 6-31 \mathrm{G} * \star / / \mathrm{HF}=-502.721747, \mathrm{G}=-22.5035 ; \lambda=-560.93,68.67$}

$\begin{array}{llllllll}0 & 0.000000 & 0.000000 & 0.000000 & \text { H } & 4.477680 & -2.199270 & -0.662690\end{array}$

$\begin{array}{lllllllll}\text { C } & 1.189140 & 0.000000 & 0.000000 & \text { H } & 5.115550 & -1.416850 & 0.774400\end{array}$

C $1.793240 \quad 2.102450 \quad 0.000000 \quad$ C $\quad 6.009360 \quad-0.699340-1.121380$

$\mathrm{H} \quad 0.956390 \quad 2.590240 \quad 0.487140 \quad \mathrm{H} \quad 6.800200 \quad-0.303700 \quad-0.480310$

$\mathrm{H} \quad 1.671270 \quad 1.944790-1.069950 \quad \mathrm{H} \quad 6.464200-1.489710-1.725490$

C $3.043020 \quad 2.129050 \quad 0.569040 \quad$ C $5.411650 \quad 0.423320 \quad-2.007970$

H $\quad 3.135330 \quad 2.556710 \quad 1.567140 \quad \mathrm{H} \quad 6.110250 \quad 1.253290-2.161660$

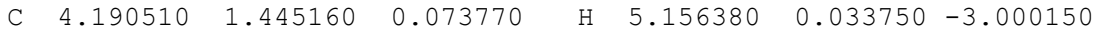

C $5.499920 \quad 1.645270 \quad 0.817500$ C $4.115380 \quad 0.852810-1.322580$

C $3.688460-0.233010-0.398800 \quad \mathrm{H} \quad 3.380610 \quad 1.318110-1.980040$

C $2.365610-0.687600-0.066780$ H $\quad 6.290170 \quad 2.031730 \quad 0.164410$

H $\quad 2.299760-1.639490 \quad 0.459790 \quad \mathrm{H} \quad 5.878980 \quad 0.730060 \quad 1.286050$

C $4.831960-1.239330-0.270640$ H $5.358000 \quad 2.375080 \quad 1.619860$

1-substituted ketene trans transition state

B3LYP/6-31G**//HF=-502.718422, G=-22.2046; $\lambda=-200.9,94.51$

C $0.000000 \quad 0.000000 \quad 0.000000 \quad$ H $3.184530 \quad 1.266040 \quad 0.190250$

$\begin{array}{llllllll}\text { C } & 1.435040 & 0.000000 & 0.000000 & \text { H } & 1.969950 & -0.865810 & -0.386770\end{array}$

$\begin{array}{llllllll}\text { C } & 2.113210 & 1.267610 & 0.000000 & \text { H } & 0.464250 & 1.853510 & -3.051870\end{array}$

C $\quad 1.985080 \quad 1.517510-1.630420 \quad$ H $-3.274520 \quad 0.471360-0.191820$

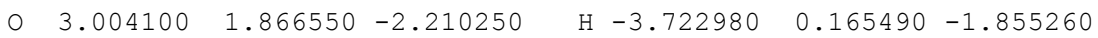

C $-0.440690 \quad 0.687460-1.484910 \quad \mathrm{H}-1.973700 \quad 1.539480 \quad-2.690210$

C $0.676810 \quad 1.287970-2.145120 \quad \mathrm{H}-1.946590 \quad 2.229520-1.077880$

$\begin{array}{lllllll}\mathrm{C}-1.841750 & 1.288500 & -1.630300 & \mathrm{H} & -0.074800 & 1.199810 & 1.792000\end{array}$

C $-2.867710 \quad 0.221200-1.175440 \quad \mathrm{H}-0.999190-0.299110 \quad 1.888330$

$\begin{array}{llllllll}\text { C } & -0.730520 & 0.532420 & 1.226980 & \mathrm{H} & -1.643160 & 1.083400 & 0.996440\end{array}$

C $-0.621090-0.792990-1.092460 \quad$ H $-2.304180-1.724700-2.016180$

C $-2.104440-1.131890-1.116940 \quad \mathrm{H}-2.415600-1.740160-0.260170$

$\mathrm{H} \quad 1.608960 \quad 2.078540 \quad 0.528930 \quad \mathrm{H} \quad 0.056020 \quad-1.488980-1.586750$

\section{2-substituted ketene cis transition state}

B3LYP $/ 6-31 \mathrm{G} * \star / / \mathrm{HF}=-502.732968, \mathrm{G}=-22.8127 ; \lambda=-560.34,82.57$

$\begin{array}{lllllllll}0 & 0.000000 & 0.000000 & 0.000000 & \text { H } & 4.546870 & -2.292720 & -0.585880\end{array}$

C $1.197020 \quad 0.000000 \quad 0.000000 \quad$ H $\quad 4.893490 \quad-1.669930 \quad 1.026290$

C $1.821350 \quad 1.950390 \quad 0.000000 \quad$ C $\quad 6.092460 \quad-0.750970-0.597640$

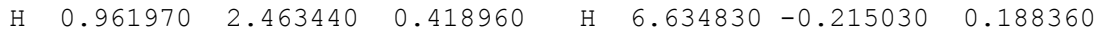

$\mathrm{H} \quad 1.814120 \quad 1.871280-1.085670 \quad \mathrm{H} \quad 6.782010-1.499560-0.997950$

$\begin{array}{llllllll}\text { C } & 3.032080 & 1.968890 & 0.675010 & \text { C } & 5.601870 & 0.237200 & -1.683820\end{array}$

$\begin{array}{llllllll}\text { C } & 3.095310 & 2.471570 & 2.095200 & \text { H } & 6.286870 & 1.081010 & -1.824920\end{array}$

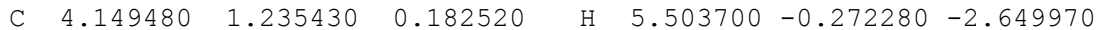

$\mathrm{H} \quad 5.071900 \quad 1.285980 \quad 0.752890 \quad \mathrm{C} \quad 4.214200 \quad 0.667880-1.215480$

C $3.691180-0.365280-0.274120 \quad$ H $3.554110 \quad 1.113540-1.957580$

$\begin{array}{lllllll}\text { C } \quad 2.322550 & -0.778020 & -0.062780 & \text { H } & 4.078290 & 2.890590 & 2.331240\end{array}$

$\mathrm{H} \quad 2.172510-1.769240 \quad 0.360410 \quad \mathrm{H} \quad 2.921090 \quad 1.638820 \quad 2.791100$

C $4.804780-1.383760-0.027520 \quad \mathrm{H} \quad 2.334190 \quad 3.231250 \quad 2.289000$

2-substituted ketene trans transition state

B3LYP / 6-31G**//HF=-502.726707, G=-22.1006; $\lambda=-358.99,87.29$

C $0.000000 \quad 0.000000 \quad 0.000000 \quad$ H $3.082490 \quad 1.390690 \quad 0.208480$

C $1.415160 \quad 0.000000 \quad 0.000000 \quad$ H $2.879110-1.475300 \quad 0.505380$

C $2.015150 \quad 1.322140 \quad 0.000000 \quad \mathrm{H} \quad 2.966940 \quad-0.952910-1.172900$

C $2.267170-1.187280-0.360950$ H $\quad 1.674330-2.058940-0.647600$

$\begin{array}{llllllll}\text { C } & -0.568390 & 0.784690 & -1.490170 & \text { H } & 0.316160 & 2.082300 & -2.941440\end{array}$

C $0.538200 \quad 1.409130-2.113490 \quad \mathrm{H}-2.287000 \quad 1.519630 \quad-2.518000$

C $1.875840 \quad 1.547230-1.614430 \quad \mathrm{H}-1.949810 \quad 2.440360-1.054200$

$\begin{array}{llllllll}\mathrm{O} & 2.901850 & 1.818590 & -2.227010 & \mathrm{H} & -0.530730 & 0.582130 & 0.749750\end{array}$

C $-1.957650 \quad 1.429290-1.473280 \quad \mathrm{H}-0.182410-1.410810-1.644170$

C $-0.754310-0.684400-1.070940 \quad \mathrm{H}-2.626340-1.375820-1.853360$

C $-2.250150-0.937890-0.921260 \quad$ H $-2.483430-1.638200-0.112380$

$\begin{array}{llllllll}\text { C }-2.872520 & 0.459920 & -0.697370 & \mathrm{H} & -2.865170 & 0.711960 & 0.369120\end{array}$

$\mathrm{H} \quad 1.447850 \quad 2.088210 \quad 0.533200 \quad \mathrm{H}-3.915010 \quad 0.507230-1.024860$ 





\# Ball-and-stick figure

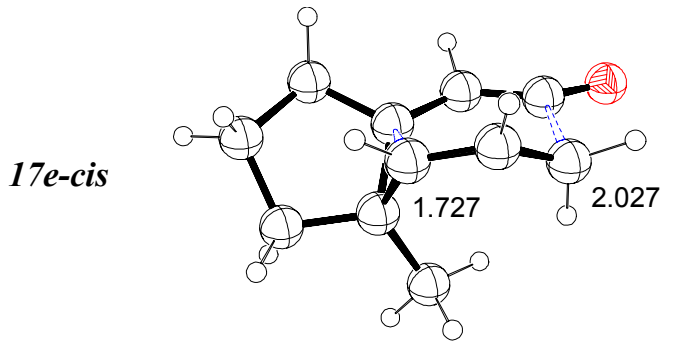

$17 e-$ trans

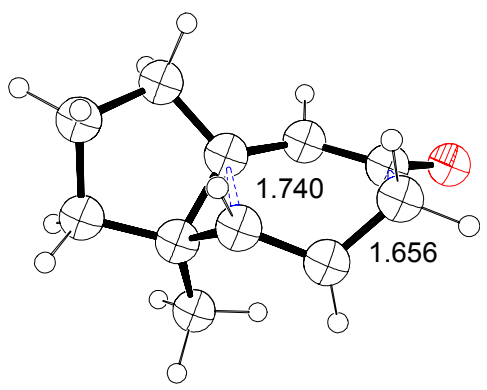

18

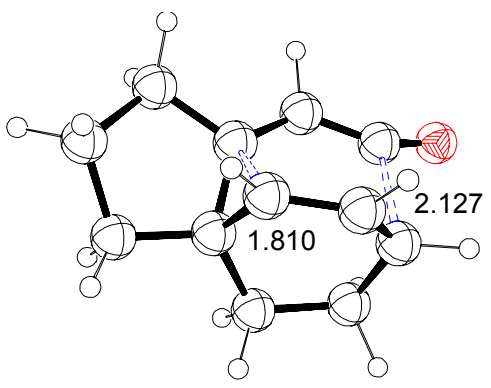

19

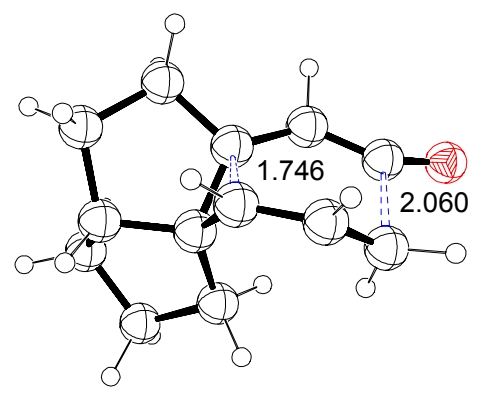

Level of theory/basis//HF (hartree), $\Delta \mathrm{G}$ correction $(\mathrm{kcal} / \mathrm{mol})$ from calc. real frequencies; $\lambda_{1}, \lambda_{2}$ (two lowest eigenvalues, transition structures) 
\# Ball-and-stick figure

20-cis

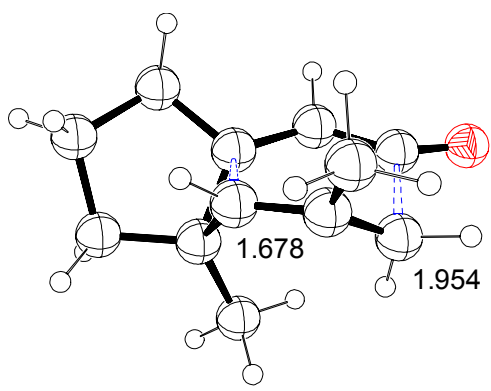

20-trans

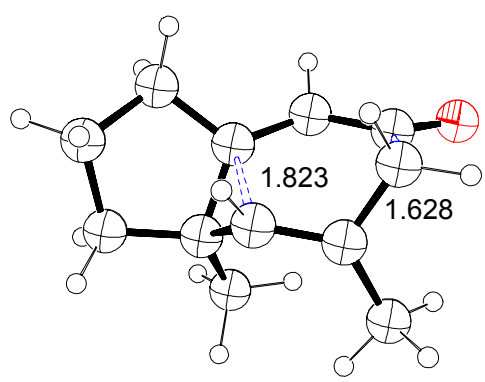

21-cis

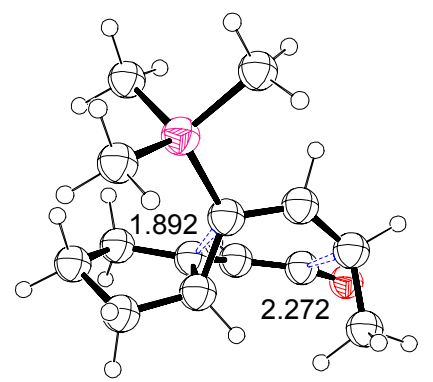

Level of theory $/$ basis $/ \mathrm{HF}$ (hartree), $\Delta \mathrm{G}$ correction $(\mathrm{kcal} / \mathrm{mol}$ ) from calc. real frequencies; $\lambda_{1}, \lambda_{2}$ (two lowest eigenvalues, transition structures)

\begin{tabular}{|c|c|c|c|c|c|c|c|}
\hline \multicolumn{8}{|c|}{ 2,5 CH3-substituted ketene cis transition state } \\
\hline \multicolumn{8}{|c|}{ B3LYP $/ 6-31 G * * / / H F=-542.045399, G=-23.8314 ; \lambda=-521.31,56.98$} \\
\hline 0 & .000000 & 0.000000 & 0.000000 & $\mathrm{H}$ & 4.108710 & -3.141880 & -1.915380 \\
\hline C & 1.203510 & 0.000000 & 0.000000 & $\mathrm{H}$ & 3.102810 & -1.879840 & -2.625300 \\
\hline C & 2.289410 & 0.839790 & 0.000000 & $\mathrm{H}$ & 2.340910 & -3.329790 & -1.949720 \\
\hline C & 3.701320 & 0.516250 & 0.101300 & $\mathrm{H}$ & 5.044570 & -1.198410 & -0.748410 \\
\hline C & 4.164970 & -1.081280 & -0.121960 & $\mathrm{H}$ & 4.495270 & 2.490780 & 044430 \\
\hline C & 3.060370 & -1.912670 & -0.478650 & $\mathrm{H}$ & 4.623230 & 1.609590 & -1.566340 \\
\hline C & 1.830420 & -1.842920 & 0.168660 & $\mathrm{H}$ & 6.542880 & 0.361200 & -0.787630 \\
\hline C & 3.154980 & -2.615220 & -1.810480 & $\mathrm{H}$ & 6.804420 & 1.829880 & 0.133220 \\
\hline C & 4.711350 & 1.506190 & -0.479310 & $\mathrm{H}$ & 6.550780 & -0.584560 & 590 \\
\hline C & 6.098730 & 1.009150 & -0.024800 & $\mathrm{H}$ & 5.878900 & 0.891160 & 2.142040 \\
\hline C & 4.380970 & -0.296760 & 1.171840 & $\mathrm{H}$ & 3.766420 & -1.757020 & 9020 \\
\hline C & 5.825020 & 0.220460 & 1.275620 & $\mathrm{H}$ & 4.226500 & -0.173850 & 3.311200 \\
\hline C & 3.717410 & -0.680640 & 2.485060 & $\mathrm{H}$ & 2.670900 & -0.363910 & 2.515950 \\
\hline & 1.004750 & -2.434000 & -0.215340 & $\mathrm{H}$ & 2.052110 & 590 & \\
\hline & 1.807 & -1.697330 & 1.243700 & & & & \\
\hline \multicolumn{8}{|c|}{ 2,5 CH3-substituted ketene trans transition state } \\
\hline \multicolumn{8}{|c|}{ B3LYP $/ 6-31 G * * / / H F=-542.040651, G=-22.8283 ; \lambda=-353.92,80.54$} \\
\hline $\mathrm{C}$ & 0.000000 & 0.000000 & 0.000000 & $\mathrm{H}$ & 2.947420 & -1.353120 & 0.588570 \\
\hline $\mathrm{C}$ & 1.411110 & 0.000000 & 0.000000 & $\mathrm{H}$ & 2.988800 & -0.970570 & -1.127310 \\
\hline $\mathrm{C}$ & 2.001960 & 1.331030 & 0.000000 & $\mathrm{H}$ & 1.761650 & -2.094270 & -0.485070 \\
\hline $\mathrm{C}$ & 1.859240 & 1.555260 & -1.605920 & $\mathrm{H}$ & 0.289570 & 2.224780 & -2.859620 \\
\hline 0 & 2.882 & 510 & -2 & $\mathrm{H}$ & -2.72 & 3180 & 730 \\
\hline $\mathrm{C}$ & 2.309880 & -1.1 & -0.288200 & $\mathrm{H}$ & -3.919580 & 0.826810 & -0 . \\
\hline $\mathrm{C}$ & -0.616190 & 0.893660 & -1.464960 & $\mathrm{H}$ & -2.375100 & 1.688980 & -2.3 \\
\hline $\mathrm{C}$ & 0.500950 & 1.490610 & -2.081530 & $\mathrm{H}$ & -1.867660 & 2.635270 & -0.982410 \\
\hline $\mathrm{C}$ & -1.961970 & 1.614160 & -1.364490 & $\mathrm{H}$ & 0.692120 & -1.414970 & -2.369600 \\
\hline C & -2.8 & 580 & -0 . & $\mathrm{H}$ & -0 . & 190 & \\
\hline C & -0.8 & -0.5 & -1.069790 & $\mathrm{H}$ & -0.27 & 7940 & -1 . \\
\hline C & -0.307150 & -1.657240 & -2.008090 & $\mathrm{H}$ & -2.849670 & -1.127350 & -1.636690 \\
\hline C & -2.346500 & -0.713920 & -0.754490 & $\mathrm{H}$ & -2.538150 & -1.398330 & 0.078670 \\
\hline $\mathrm{H}$ & 1.428750 & 2.092710 & 0.533710 & $\mathrm{H}$ & -0.500780 & 0.610960 & 0.749430 \\
\hline & 3.067 & 3150 & 0.216670 & & & & \\
\hline & $\left(\mathrm{Si}(\mathrm{CH} 3)_{3}\right)$ & $4-($ CHO $)$ &  & & cis & ition & te \\
\hline \multicolumn{8}{|c|}{ B3LYP $/ 6-31 \mathrm{G} * \star / / \mathrm{HF}=-911.389283, \mathrm{G}=-28.4075 ; \lambda=-536.77,40.82$} \\
\hline 0 & 0.0 & 00 & 0 . & $\mathrm{H}$ & 60 & 520 & 80 \\
\hline $\mathrm{C}$ & 1.186880 & 000 & 0.000000 & $\mathrm{H}$ & 490 & 9960 & 60 \\
\hline $\mathrm{C}$ & 2.367120 & 0.686130 & 0.000000 & $\mathrm{H}$ & 1.679590 & -3.087430 & 2.241310 \\
\hline $\mathrm{C}$ & 3.685080 & 0.296080 & 0.398900 & $\mathrm{H}$ & 0.191940 & -2.250990 & 1.774130 \\
\hline $\mathrm{C}$ & 4.800270 & 1.321050 & 0.199300 & $\mathrm{H}$ & 0.963910 & -2.638340 & -0.382010 \\
\hline C & 6.00 & 0.8 & 1.014 & $\mathrm{H}$ & 850 & -2.529990 & -1.318340 \\
\hline C & 4.126 & -0.7 & 1.413290 & $\mathrm{H}$ & 040 & 5660 & -2.22 \\
\hline C & 4.185100 & -1.507110 & 0.119910 & $\mathrm{H}$ & 4.539810 & -3.534080 & -2.574660 \\
\hline C & 2.979190 & -2.154550 & -0.296160 & $\mathrm{H}$ & 4.886420 & -4.440270 & -1.096350 \\
\hline $\mathrm{C}$ & 1.716140 & -2.193420 & 0.266880 & $\mathrm{H}$ & 7.015540 & -1.316640 & -2.856770 \\
\hline $\mathrm{C}$ & 1.282820 & -2.203550 & 1.726370 & $\mathrm{H}$ & 6.692410 & 0.079790 & -1.826610 \\
\hline $\mathrm{Si}$ & 5.718180 & -2.070600 & -0.870030 & $\mathrm{H}$ & 5.411530 & -0.575980 & -2.852900 \\
\hline C & 5.278100 & -3.679050 & -1.779210 & $\mathrm{H}$ & 7.962680 & -3.000730 & -0.367520 \\
\hline $\mathrm{C}$ & 6.260800 & -0.846490 & -2.215380 & $\mathrm{H}$ & 6.894010 & -3.226270 & 1.019390 \\
\hline $\mathrm{C}$ & 7.192050 & -2.513740 & 0.242160 & $\mathrm{H}$ & 7.659480 & -1.657520 & 0.735120 \\
\hline $\mathrm{C}$ & 5.415620 & -0.168900 & 2.044640 & $\mathrm{H}$ & 5.141110 & 0.367900 & 2.961070 \\
\hline $\mathrm{H}$ & 6.728900 & 0.314170 & 0.364640 & $\mathrm{H}$ & 6.113630 & -0.960090 & 2.333380 \\
\hline $\mathrm{H}$ & 6.550580 & 1.638080 & 1.495640 & $\mathrm{H}$ & 3.411750 & -1.061550 & 2.147250 \\
\hline H & 4.437290 & 2.271530 & 0.611470 & $\mathrm{H}$ & 2.294500 & 1.583610 & -0.615210 \\
\hline
\end{tabular}




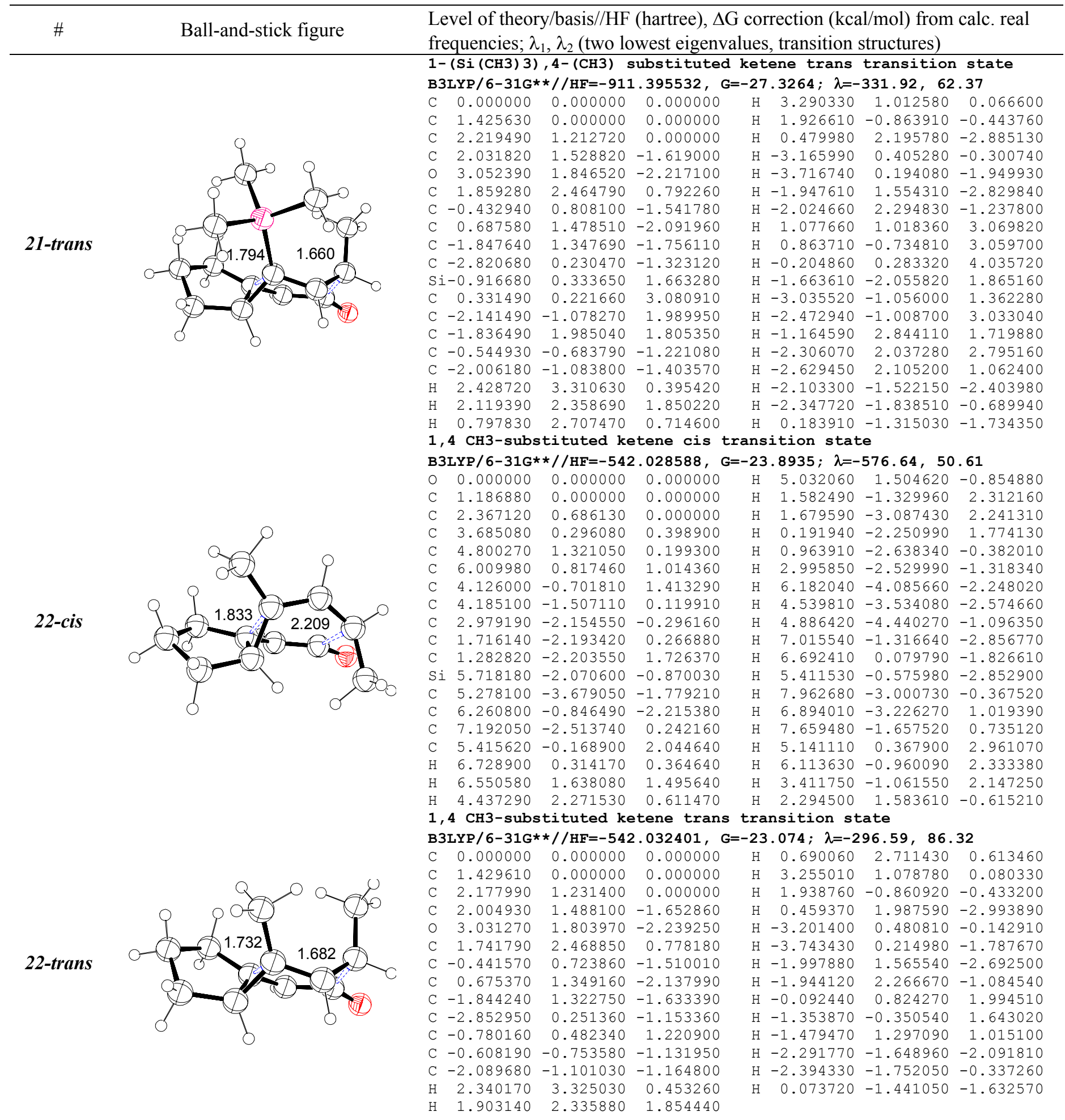




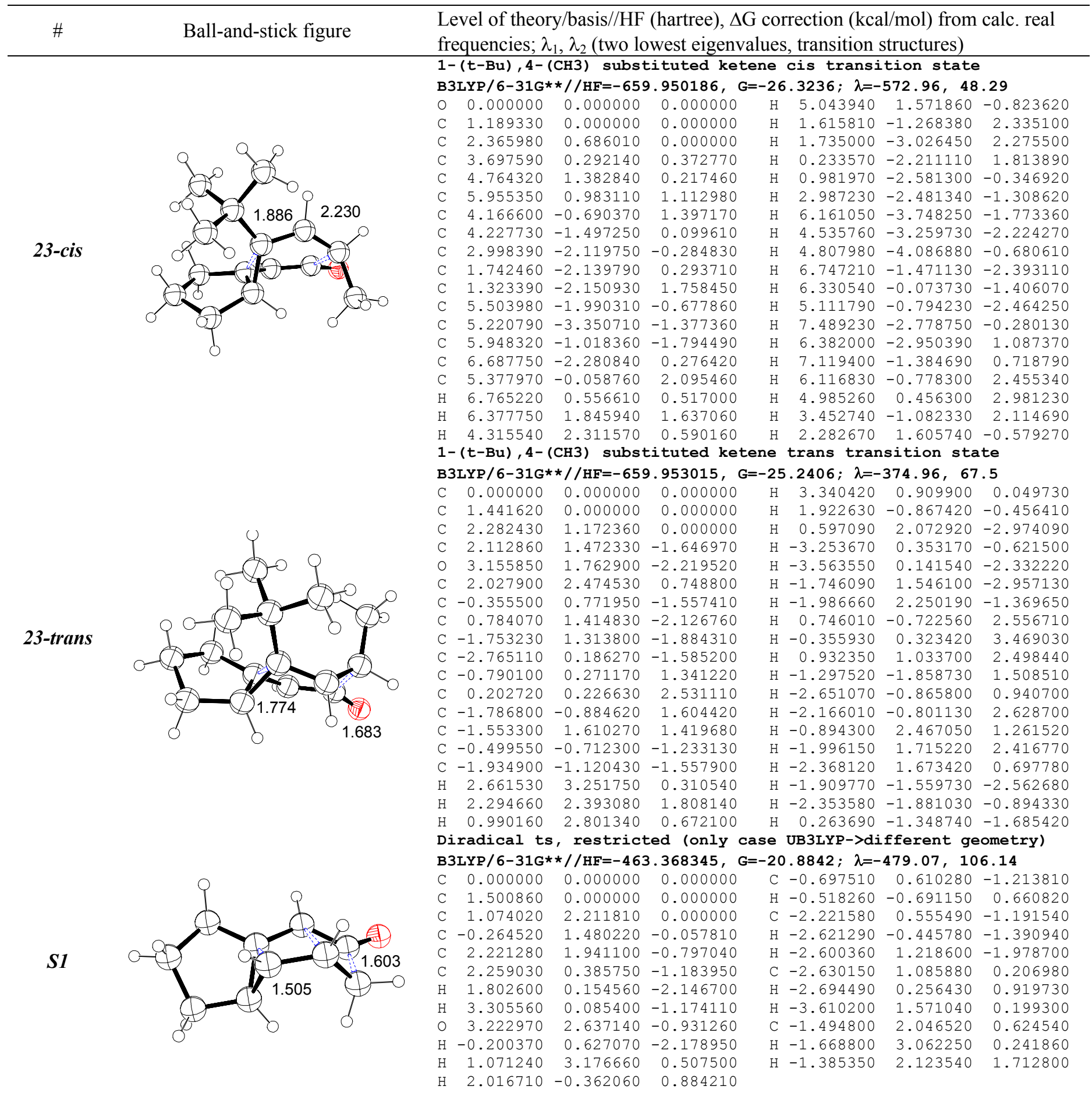


\# Ball-and-stick figure

$S 2$

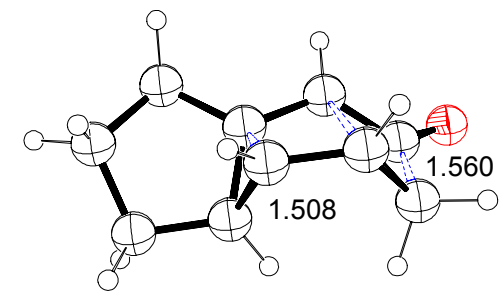

S3



$S 4$

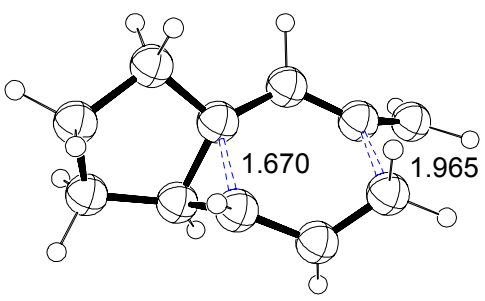

S5

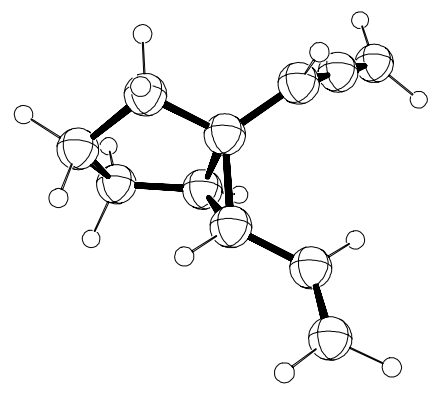

Level of theory $/$ basis $/ \mathrm{HF}$ (hartree), $\Delta \mathrm{G}$ correction $(\mathrm{kcal} / \mathrm{mol})$ from calc. real

frequencies; $\lambda_{1}, \lambda_{2}$ (two lowest eigenvalues, transition structures)

\section{UB3LYP $/ 6-31 \mathrm{G} * \star / / H F=-463.369972, G=-20.9642 ; \lambda=-627.18,93.38$}

$\begin{array}{llllllll}C & 0.000000 & 0.000000 & 0.000000 & C & -0.685780 & 0.599990 & -1.214590\end{array}$

$\begin{array}{llllllll}\text { C } & 1.509910 & 0.000000 & 0.000000 & \text { H } & -0.523680 & -0.697740 & 0.650180\end{array}$

$\begin{array}{llllllll}\text { C } & 1.079630 & 2.173260 & 0.000000 & \text { C } & -2.210830 & 0.553340 & -1.215900\end{array}$

C $-0.273740 \quad 1.482350-0.052390 \quad \mathrm{H}-2.610930-0.448690-1.410280$

C $2.194660 \quad 1.935340-0.898360 \quad \mathrm{H}-2.574670 \quad 1.208780 \quad-2.016420$

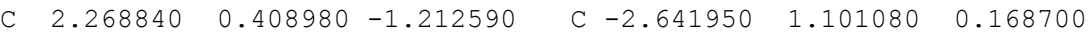

H $\quad 1.830750 \quad 0.106250-2.166830 \quad \mathrm{H}-2.713810 \quad 0.280710 \quad 0.891190$



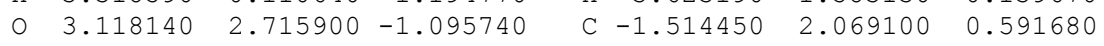

$\mathrm{H}-0.178370-0.629270-2.173930-\mathrm{H}-1.682370-3.077150-0.186860$

$\mathrm{H} \quad 1.141230 \quad 3.088450 \quad 0.589750 \quad \mathrm{H}-1.430530 \quad 2.165290 \quad 1.680810$

$\mathrm{H} \quad 2.031200-0.405280 \quad 0.861440$

Allyl-substituted cis transition state

B3LYP $/ 6-31 G * \star / / H F=-427.472647, G=-21.539 ; \lambda=-506.88,87.77$

C $0.000000 \quad 0.000000 \quad 0.000000 \quad \mathrm{H}-0.136140-3.305240 \quad 0.796960$

C $2.318620 \quad 0.000000-0.000000 \quad$ C $\quad 1.035530-1.926130 \quad 2.070300$

$\begin{array}{lllllllll}\text { C } & 2.647040 & 1.277660 & 0.000000 & \text { H } & 0.962670 & -0.903720 & 2.439970\end{array}$

$\mathrm{H} \quad 1.987380 \quad 2.055570-0.374770 \quad \mathrm{C} \quad 1.167880 \quad-3.014000 \quad 3.137430$

$\mathrm{H} \quad 3.601290 \quad 1.608460 \quad 0.408860 \quad \mathrm{H} \quad 1.872880 \quad-2.671130 \quad 3.903970$

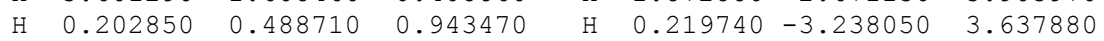

$\begin{array}{llllllll}\mathrm{H} & -0.305560 & 0.659100 & -0.806820 & \mathrm{C} & 1.746940 & -4.234630 & 2.383770\end{array}$

C $-0.322220-1.337700-0.035620$ H $2.330950-4.886850 \quad 3.039150$



C $2.669800-1.307670 \quad 0.135700$ C $2.600050-3.635040 \quad 1.240950$

H $\quad 3.307480-1.732850-0.642530 \quad$ H $2.591160 \quad-4.253470 \quad 0.336410$

C $2.055860-2.229630 \quad 1.014720$ H $3.648670-3.549300 \quad 1.553530$

C $0.176600-2.270800 \quad 0.893020$

Allyl-substituted trans transition state

B3LYP / 6-31G* $/ / / H F=-427.458735, G=-21.479 ; \lambda=-497.79,93.03$

C $0.000000 \quad 0.000000 \quad 0.000000 \quad$ C $1.264310-2.584510 \quad 2.109910$

C $\quad 1.965350 \quad 0.000000 \quad 0.000000 \quad \mathrm{H} \quad 1.614300-1.838010 \quad 2.821180$

C $\quad 2.467530 \quad 1.234170 \quad 0.000000 \quad$ H $-0.465280 \quad-2.837620 \quad 0.707060$

$\begin{array}{llllllll}\mathrm{H} & 3.475290 & 1.431540 & 0.355620 & \mathrm{C} & 1.371920 & -4.052680 & 2.490080\end{array}$

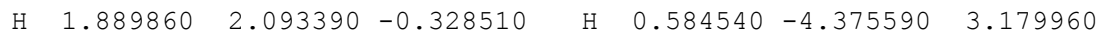

C $-0.129010-0.718850 \quad 1.185950 \quad \mathrm{H} \quad 2.333890 \quad-4.218090 \quad 2.990020$

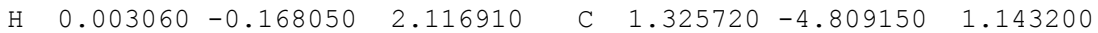

$\mathrm{H}-0.198430-0.504810-0.943120 \quad \mathrm{H} \quad 0.287320 \quad-5.024620 \quad 0.869620$

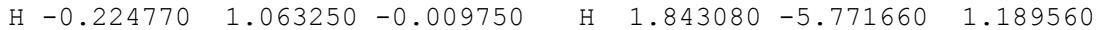

C $2.358680-1.322500-0.008310$ C $1.959370-3.845720 \quad 0.116740$

$\mathrm{H} \quad 3.050860-1.597740-0.805220 \quad \mathrm{H} \quad 1.550070 \quad-3.981780 \quad-0.890560$

C $\quad 1.738020-2.428610 \quad 0.658550 \quad$ H $3.041260-4.016200 \quad 0.046870$

C $0.187710-2.120260 \quad 1.197400$

Allyl-substituted starting structure

B3LYP / 6-31G**//HF $=-427.497237, G=-22.917$

C $0.000000 \quad 0.000000 \quad 0.000000$

$\begin{array}{llll}\text { C } \quad 4.841130 & 0.000000 & 0.000000\end{array}$

$\begin{array}{llll}\text { C } & 5.538960 & 1.105810 & 0.000000\end{array}$

H $\quad 6.569710 \quad 1.130010 \quad 0.348130$

$\mathrm{H} \quad 5.118110 \quad 2.046290 \quad-0.350610$

C $1.273300-0.045560 \quad 0.400980$

$\begin{array}{llll}\mathrm{H} & 1.968320 & 0.716200 & 0.053100\end{array}$

$\mathrm{H}-0.726960 \quad-0.742440 \quad 0.320520$

$\mathrm{H}-0.360600 \quad 0.782360-0.660720$

$\begin{array}{llll}\text { C } \quad 4.124790 & -1.097040 & 0.008460\end{array}$

$\mathrm{H} \quad 4.139620-1.737490-0.875190$

C $3.281380-1.560180 \quad 1.151450$

C $1.828310-1.074790 \quad 1.302750$
C $2.896290 \quad-0.692620 \quad 2.319690$

H $\quad 3.202420 \quad 0.349400 \quad 2.349430$

H $1.103230-1.827340 \quad 1.608400$

C $2.978120-1.521730 \quad 3.593950$

H $2.180870-1.294030 \quad 4.310870$

H $3.932970-1.312910 \quad 4.092550$

C $2.930090-2.990720 \quad 3.109130$

H $\quad 1.893070-3.338750 \quad 3.063840$

$\mathrm{H} \quad 3.458390-3.671170 \quad 3.783310$

C $3.550810-2.969400 \quad 1.693100$

$\mathrm{H} \quad 3.152760-3.760880 \quad 1.046930$

H $\quad 4.637140 \quad-3.111120 \quad 1.749270$ 


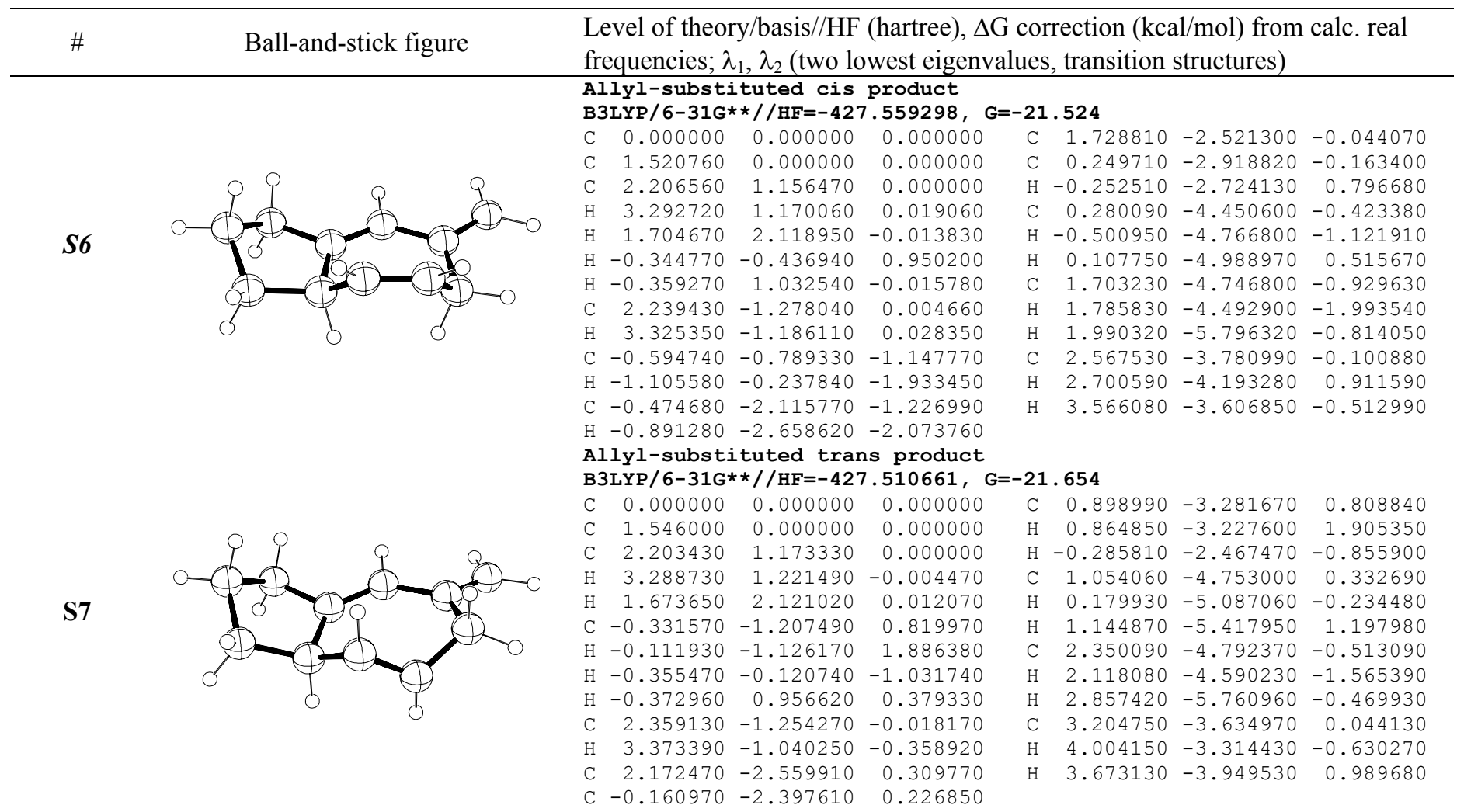

Note on restricted/unrestricted calculations: All transition states were calculated using closed shell methods, then reoptimized with unrestricted DFT with appropriate combinations of orbitals taken to break spin symmetry. In all transition states but one (S1), the energy and geometry remain unchanged and this is indicated with the notation (UB3LYP $\rightarrow$ closed shell) in the table above. In structure S1, the energy and geometry does change, and this is indicated with the note (UB3LYP $\rightarrow$ different geometry) in the table above. In that case the unrestricted energy and geometry is shown in addition to the restricted energy and geometry. 